OPEN ACCESS

Edited by:

Jun-Lin Yi,

Chinese Academy of Medical

Sciences and Peking Union

Medical College, China

Reviewed by:

Jiamei Fu,

Shanghai Pulmonary Hospital,

China

Hamid Mammar,

Institut Curie, France

*Correspondence:

Arnab Pal

pal.arnab@pgimer.edu.in

${ }^{\dagger}$ These authors have contributed equally to this work and share first authorship

${ }^{\ddagger}$ These authors share senior authorship

Specialty section: This article was submitted to Radiation Oncology, a section of the journal

Frontiers in Oncology

Received: 11 January 2021 Accepted: 28 April 2021

Published: 17 May 2021

Citation:

Gupta A, Mathew D, Bhat $S A$,

Ghoshal $S$ and $\mathrm{Pal} A$ (2021)

Genetic Variants of DNA

Repair Genes as Predictors of Radiation-Induced Subcutaneous Fibrosis in Oropharyngeal Carcinoma

Front. Oncol. 11:652049.

doi: 10.3389/fonc.2021.652049

\section{Genetic Variants of DNA Repair Genes as Predictors of Radiation- Induced Subcutaneous Fibrosis in Oropharyngeal Carcinoma}

\author{
Ankita Gupta $^{1 \dagger}$, Don Mathew ${ }^{2 \dagger}$, Shabir Ahmad Bhat ${ }^{2}$, Sushmita Ghoshal ${ }^{1 \neq}$ and Arnab Pal ${ }^{2 \not \neq}$ \\ ${ }^{1}$ Department of Radiotherapy, Post Graduate Institute of Medical Education and Research, Chandigarh, India, \\ 2 Department of Biochemistry, Post Graduate Institute of Medical Education and Research, Chandigarh, India
}

Purpose: To investigate the impact of genetic variants of DNA repair and pro-fibrotic pathway genes on the severity of radiation-induced subcutaneous fibrosis in patients of oropharyngeal carcinoma treated with radical radiotherapy.

Materials and Methods: Patients of newly diagnosed squamous cell carcinoma of oropharynx being treated with two-dimensional radical radiotherapy were enrolled in the study. Patients who had undergone surgery or were receiving concurrent chemotherapy were excluded. Patients were followed up at 6 weeks post completion of radiotherapy and every 3 months thereafter for a median of 16 months. Subcutaneous fibrosis was graded according to the Radiation Therapy Oncology Group (RTOG) and the European Organization for Research and Treatment of Cancer (EORTC) grading system and the maximum grade was recorded over the length of the patient's follow-up. Patients with severe fibrosis ( $\geq G 3)$, were compared to patients with minor $(\leq \mathrm{G} 2)$ fibrotic reactions. Eight single nucleotide polymorphisms of 7 DNA repair genes and 2 polymorphisms of a single pro-fibrotic pathway gene were analyzed by Polymerase Chain Reaction and Restriction Fragment Length Polymorphism and were correlated with the severity of subcutaneous fibrosis.

Results: 179 patients were included in the analysis. Subcutaneous fibrosis was seen in 168 (93.9\%) patients. 36 (20.1\%) patients had severe (grade 3) fibrosis. On multivariate logistic regression analysis, Homozygous CC genotype of XRCC3 (722C>T, rs861539) $\left(p=0.013^{*}\right.$, OR 2.350, 95\% Cl 1.089-5.382), Homozygous AA genotype of ERCC4 Ex8 $\left(1244 \mathrm{G}>\mathrm{A}, \mathrm{rs1800067)}\left(p=0.001^{* *}, \mathrm{OR} 11.626,95 \% \mathrm{Cl} 2.490-275.901\right)\right.$ and Homozygous TT genotype of XRCC5 (1401G>T, rs828907) $\left(p=0.020^{*}\right.$, OR 2.188, 95\% $\mathrm{Cl} 1.652-7.334)$ were found to be predictive of severe subcutaneous fibrosis. On haplotype analysis, the cumulative risk of developing severe fibrosis was observed in patients carrying both haplotypes of variant Homozygous AA genotype of ERCC4 Ex8 (1244G>A, rs1800067) and Homozygous TT genotype of XRCC5 (1401 G>T, rs828907) $\left(p=0.010^{*}\right.$, OR $\left.26.340,95 \% \mathrm{Cl} 4.014-76.568\right)$. 
Conclusion: We demonstrated significant associations between single nucleotide polymorphisms of DNA repair genes and radiation-induced subcutaneous fibrosis in patients of oropharyngeal carcinoma treated with radiotherapy. We propose to incorporate these genetic markers into predictive models for identifying patients genetically predisposed to the development of radiation-induced fibrosis, thus guiding personalized treatment protocols.

Keywords: single nucleotide polymorphisms (SNPs), subcutaneous fibrosis, radiation-induced toxicity, DNA repair genes, oropharyngeal carcinoma

\section{INTRODUCTION}

India has the highest incidence rate of oropharyngeal carcinoma (OPC) in the world, with majority $(68.6 \%)$ of the patients presenting in locoregionally advanced stages of the disease (1). Radiotherapy with concurrent chemotherapy has been the standard non-surgical treatment for locally advanced OPC (2). However the toxicity of intensive treatment regimens also contributes to a substantial increase in patient morbidity and mortality, especially in developing countries like India, with patient profiles distinct from the western world. Most patients present with poor performance and nutritional status and inadequate support systems. This leads to poor compliance and treatment tolerability and hence, poor disease outcomes. Hence, majority of our patients receive definitive radiotherapy alone with conventional or altered fractionation schedules in order to achieve acceptable outcomes with minimum morbidity. Moreover, the enormous patient load in high volume referral centers imparts greater logistic difficulties in devoting the time and infrastructure to execute conformal treatment planning for every patient (3-5). Therefore, 2-dimensional conventional radiotherapy continues to be used for a significant proportion of our patients $(6,7)$.

The treatment fields used in conventional radiotherapy for OPC include large volumes of the oral cavity, pharynx and the neck resulting in high predisposition to radiation induced normal tissue toxicity (8). While acute radiotoxicities interrupt the routine treatment schedule and limit the radiation dose, long-term radiotoxicities significantly impair the quality of life of these patients (5). The most frequently encountered acute radiotoxicities in OPC are oral mucositis, dermatitis and dysphagia; while delayed toxicities include late-onset xerostomia, fibrosis and rarely, osteoradionecrosis of the mandible $(9,10)$.

Radiation-induced subcutaneous fibrosis, a late radiotoxicity response, results from dysregulation of inflammation and regeneration. It is one of the most common long-term toxicities of head and neck cancer (HNC) therapy and has been reported in more than $70 \%$ of the patients at some point after HNC treatment, causing cosmetic and functional impairment that significantly impacts quality of life (11-13). A number of factors increase the risk of radiation-induced fibrosis. These factors are treatment related (total dose, dose per fraction, volume irradiated, irradiation site and dose inhomogeneity, additional treatment like use of concomitant chemotherapy or surgery) or patient-specific (age, smoking, alcohol and tobacco usage and co-morbid conditions such as diabetes, vascular and connective tissue disorders) $(11,12)$.
However even with uniform treatment protocols, not all patients develop subcutaneous fibrosis and other radiotoxicities of the same severity. Apart from patient-specific factors, almost $80 \%$ of this inter-individual variability has been attributed to genetic differences amongst individuals $(14,15)$. The genetic pathways involved in radiation response(s) encompass a multitude of genes involved in processes such as DNA double-strand break (DSB) repair, DNA damage response, cell-cycle control, apoptosis, cellular antioxidant defenses and fibrosis (14-16).

Single nucleotide polymorphisms (SNPs) are DNA sequence variations that arise when a single nucleotide within a gene is altered. SNPs constitute more than $99 \%$ of all genetic variations that can affect mRNA stability, rates of transcription, protein translation and/or regulation of gene methylation resulting in dysregulated function and varying degrees of clinical radiosensitivity $(14,17)$. After a thorough literature search for identifying candidate genetic polymorphisms, we selected 7 genes related to DNA repair and one from the pro-fibrotic pathway for their presumed or demonstrated role in radiosensitivity. We hypothesize that SNPs in one or more genes involved in the above radiation response pathways can interfere with their function and trigger the development of radiotherapy induced normal tissue toxicity (16).

A multitude of DNA repair pathways are activated in response to radiation induced DNA damage. DNA double strand break (DSB) repair pathways include Homologous Recombination (HR) and Non-Homologous End Joining (NHEJ) repair. These are of critical importance in the repair of DNA damage that occurs in normal tissue adjoining the tumour as a result of radiation therapy. Single-stranded breaks (SSB) are repaired by Base Excision Repair (BER), Nucleotide excision repair (NER) and Mismatch repair (MMR) pathways. SNPs in DNA repair genes may alter the ability of these cells to repair radiation induced DNA damage ultimately resulting in more severe toxicity (18-20).

XRCC1 i.e. X-Ray repair cross complimenting 1 protein participates in BER pathway of SSB caused by ionizing radiation. XRCC1 (rs25487) polymorphism is a G to A transition at codon 399 that results in change from Arg to Gln within the XRCC1 protein. The resultant protein has altered fidelity and DNA repair efficiency. Besides, carriers of XRCC1 AA genotypes have higher levels of chromosomal breaks per cell when compared with other genotypes. Genetic variants of this gene have previously been linked to worse treatment outcomes as well as increased acute and late radiotoxicity $(7,21-23)$.

XRCC3 gene, a member of Rad-51-related genes, is an indispensable component of the HR pathway of DNA DSB 
repair and inter-strand cross-links, which plays an essential role in maintaining genomic stability. Variants of XRCC3 have been shown to be positively associated with late radiation-induced toxicity and elevated cancer risk (23-25).

The ERCC4 i.e. excision repair cross-complimentary group 4 gene forms a complex with ERCC1 to encode the two subunits of the ERCC1-XPF (xeroderma pigmentosum complementation group F) nuclease. This enzyme plays a central role in NER, DNA cross-link repair and is also involved in the incision step of NHEJ repair pathway (26). ERCC4 variants have been tested for their role in influencing radiation toxicities in HNCs (27).

XRCC5 is another important component playing a crucial role in NHEJ pathway of DNA DSB repair. SNPs in XRCC5 result in major structural changes in XRCC5 protein, rendering it unavailable for the NHEJ pathway. Polymorphisms in this gene have been shown to influence cancer risk and chromosomal radiosensitivity $(28,29)$.

Rad51 (RecA homolog, Escherichia coli) protein is a component of the HR repair pathway of DNA DSBs and interstrand cross-links. Genetic variants of Rad51 influence mRNA stability and translational efficiency and have been linked to carcinogenesis and radiosensitivity (30-32).

TGF $\beta 1$ ie. transforming growth factor $\beta 1$ encodes for the versatile cytokine TGF $\beta 1$ assumed to be involved in response to tissue injuries and has been suggested to play a role in radiation response. Polymorphic variations in TGF $\beta 1$ gene can alter protein expression contributing to the initiation, development, and persistence of radiation-induced fibrosis $(33,34)$.

The association of genetic polymorphisms with late radiotoxicities has been well explored in patients of breast and prostate cancer. However, few studies have explored the correlation between genetic polymorphisms and late radiotherapy toxicity in patients with HNCs $(33,35-38)$. Moreover, no such studies have been conducted on the Indian population, which harbors the largest number of HNC patients in the world, contributing to significant cancer-related morbidity (1).

Therefore, we conducted a prospective study on a carefully selected homogeneous cohort of OPC receiving definitive radical radiotherapy by two-dimensional conventional technique to evaluate the impact of SNPs of DNA repair and pro-fibrotic pathway genes on the severity of radiation-induced subcutaneous fibrosis.

Through this radiogenomic study, we aim to identify genetic biomarkers which can be incorporated into predictive models of radiation-induced subcutaneous fibrosis. This could aid in formulation of tailored treatment regimens by identifying 'atrisk' patient groups and assigning them to treatment by more conformal radiotherapy techniques like 3-dimensional conformal (3DCRT) or Intensity Modulated Radiation Therapy (IMRT). This personalization would also allow judicious allocation of the limited available resources and help achieve better outcome with minimum morbidity.

\section{MATERIALS AND METHODS}

This prospective study was conducted at a tertiary care referral center in North India with approval from the Institutional Ethics
Committee (INT/IEC/2016/2124). Patients of newly diagnosed early inoperable and locoregionally advanced squamous cell carcinoma of the oropharynx (AJCC $7^{\text {th }}$ edition) being treated with two-dimensional radical radiotherapy were enrolled for a total period of two years and seven months. Patients who had undergone surgery or were receiving concurrent chemotherapy were excluded from our study. Those suffering from comorbidities such as diabetes mellitus, collagen vascular or immunosuppressive disorders were also excluded. Written informed consent was obtained from all study participants.

\section{Genotyping Analysis}

$5 \mathrm{ml}$ blood samples were drawn in Ethylenediaminetetraacetic acid (EDTA) vials from all recruited patients on the day of start of therapy and stored at $-20^{\circ} \mathrm{C}$. For polymorphism analysis, DNA isolation was done using the Macherey Nagel DNA isolation kit ${ }^{\mathrm{TM}}$ (GmBH, Germany) according to manufacturer's instructions.

Eight SNPs of seven DNA repair genes namely, XRCC1 (1196 $\mathrm{G}>\mathrm{A}$, rs25487), XRCC3 (722 C>T, rs861539), XRCC4 (-1394 $\mathrm{T}>\mathrm{G}, \mathrm{rs689366)}$, XRCC5 (-1401 G>T, rs828907), XRCC6 (-1310 C $>$ G, rs22677437), ERCC4Ex11 (2505 T>C, rs1799801), ERCC4Ex8 (1244 G>A, rs1800067), $\operatorname{Rad} 51$ (172 G>T, rs1801321) and two SNPs of the pro-fibrotic pathway gene i.e. TGFß1 (869 T>C, rs1982073) and TGFß1 (-509 C>T, rs1800469) were analyzed by PCR (Polymerase Chain Reaction) RFLP (Restriction Fragment Length Polymorphism) (Supplementary Data, Table 1 for PCR conditions and primer list).

\section{Treatment and Evaluation During Radiotherapy}

All patients underwent pre-treatment simulation in a fluoroscopy simulator with an immobilizing thermoplastic cast and were treated by 2-dimensional conventional planning in a telecobalt unit or low energy Linear Accelerator as per established protocols at our center. Elective nodal irradiation was performed in all patients. Bilateral parallel-opposed lateral fields were used without any tissue compensators. An additional lower anterior field was used in selected patients.

A dose of $40 \mathrm{~Gy}$ in 20 fractions was delivered to the primary and draining lymph nodes over 4 weeks (phase I), which was followed by a dose of $20 \mathrm{~Gy}$ in 10 fractions after sparing the spinal cord (phase II). An additional 6 Gy in 3 fractions (phase III) was delivered to the gross tumour with $2 \mathrm{~cm}$ margins to a total dose of 66 Gy in 33 fractions. Dose schedule of $45 \mathrm{~Gy}$ in 25 fractions over 5 weeks along with a concomitant boost of $22.5 \mathrm{~Gy}$ in 15 fractions to the gross primary and nodal disease with 1.5 to $2 \mathrm{~cm}$ margin over the last 3 weeks of treatment was used in selected patients. During treatment, patients were evaluated twice a week for acute radiation toxicities like oral mucositis, dysphagia and dermatitis.

\section{Follow-up and Toxicity Assessment}

The patients were followed up at 6 weeks post completion of radiotherapy for assessment of response and toxicity evaluation, and every 3 months thereafter. The median follow up was 16 months (range 13-48 months). Patients with a follow-up of less than 12 months were excluded. The time of development of subcutaneous fibrosis was documented. The grade of subcutaneous fibrosis was jointly evaluated by two participating 
physicians according to the Radiation Therapy Oncology Group (RTOG) and European Organization for Research and Treatment of Cancer (EORTC) grading system (39). The maximum grade of fibrosis recorded over the length of the patients' follow-up has been reported. For comparison, patients with severe fibrosis $(\geq G 3)$, referred to as the radiosensitive group (cases), were compared to the patients with minor ( $\leq \mathrm{G} 2)$ fibrotic reactions (controls).

\section{Patient-Specific Factors}

In addition, patient-specific clinical characteristics such as age, smoking habits and history of tobacco chewing and alcohol consumption etc. were also documented and analyzed in relation to the severity of subcutaneous fibrosis.

\section{Statistical Analysis}

The distribution of SNP genotypes and clinical characteristics within the radiosensitive group and the control group was analyzed by using Pearson's Chi-square test or Fisher's exact test. Binary logistic regression analysis was performed to evaluate the association of the significant variables with the risk of developing severe radiationinduced subcutaneous fibrosis. The genotypic frequencies were examined by estimating the Odd's Ratio (OR) and 95\% confidence interval (CI) of the wild, heterozygous and homozygous variant genotypes using the other two genotypes as the reference.

A multivariate logistic regression analysis was carried out to measure the independent predictive value of each SNP on the risk of severe subcutaneous fibrosis. A $p$ value of $<0.05$ was considered statistically significant.

To explore the association of the combined effect of these variants with increased risk of severe fibrosis, a haplotype association analysis was performed for two polymorphisms; ERCC4Ex8 (1244 G>A, rs1800067) and XRCC5 (-1401 G>T, rs828907). Patients were subdivided into three risk categories. Group 1 was considered as the reference category and included patients with wild type homozygous and heterozygous genotypes of both polymorphisms. Group 2 included those patients who had haplotypes containing the variant homozygous genotype of any one of the polymorphisms indicating an intermediate risk category. Group 3 included those patients who had haplotypes containing the variant homozygous genotypes of both polymorphisms indicating a high-risk category. Binary logistic regression analysis was carried out to evaluate the risk associated with the latter two groups while keeping the first group as the reference.

All the above analyses were carried out with Statistical package for Social Sciences (SPSS) version 23.

\section{RESULTS}

\section{Study Patients}

195 patients of OPC were enrolled in the study. Of these, 16 patients were excluded due to incomplete treatment and lack of follow-up. The remaining 179 patients were included for final analysis. Patient and disease characteristics have been listed in Table $\mathbf{1}$.
TABLE 1 | Patient and disease characteristics.

\begin{tabular}{lc}
\hline Mean age (years) & $58.9 \pm 8.6$ \\
\hline Gender & $\mathbf{N}(\%)$ \\
Male & \\
Female & $163(89.4 \%)$ \\
\hline Subsite & $16(8.9 \%)$ \\
Base of tongue & \\
Soft palate & $77(42.5 \%)$ \\
Tonsil & $59(32.6 \%)$ \\
Vallecula & $32(17.7 \%)$ \\
\hline Stage (WHO th $^{\text {th }}$ edition) & $13(7.2 \%)$ \\
I & \\
II & \\
III & $2(1.1 \%)$ \\
IVA & $21(11.6 \%)$ \\
IVB & $59(32.6 \%)$ \\
Smoking & $95(52.5 \%)$ \\
Tobacco chewing & $2(1.1 \%)$ \\
Alcohol intake & $160(89.4 \%)$ \\
& $28(17.2 \%)$ \\
& $104(58.1 \%)$ \\
\end{tabular}

\section{Toxicity Analysis and Association With Genotypic and Clinical Factors}

The genotypic frequencies of all SNPs and their association with radiation-induced subcutaneous fibrosis are shown in Table 2.

\section{Subcutaneous Fibrosis}

Subcutaneous fibrosis was seen in 168 (93.9\%) patients, and was more evident in the neck than the face. Thirty-six $(20.1 \%)$ patients developed grade 3 fibrosis. Grade 2 fibrosis was observed in $47(26.3 \%)$ patients. Grade 1 fibrosis was the most common and was seen in $85(47.5 \%)$ patients. Grade 4 fibrosis was not seen in any patient.

Chi-square analysis revealed significant association between the genotypic frequencies of XRCC3 $(722 \mathrm{C}>\mathrm{T}, \mathrm{rs} 861539)\left(p=0.012^{*}\right)$, ERCC4Ex8 (1244 G>A, rs1800067) $\left(p=0.003^{* *}\right)$, XRCC5 (1401 $\mathrm{G}>\mathrm{T}, \mathrm{rs} 828907)\left(p=0.046^{*}\right)$ and TGF $\beta 1(869 \mathrm{~T}>\mathrm{C}, \mathrm{rs} 1982073)$ $\left(p=0.045^{\star}\right)$ polymorphisms and severe subcutaneous fibrosis. Amongst clinical factors, history of alcohol intake showed a significant correlation $\left(p=0.017^{\star}\right)$ with fibrosis.

On univariate logistic regression analysis, following SNPs were found to be significantly associated with the risk of severe subcutaneous fibrosis; Homozygous CC genotype of XRCC3 (722 C>T, rs861539) $\left(p=0.015^{\star}\right.$, OR $2.227,95 \%$ CI $1.741-$ 6.696), Homozygous AA genotype of ERCC4Ex8 (1244 G>A, rs1800067) $\left(p=0.012^{* *}\right.$, OR 23.143, 95\% CI 1.974-271.362), Homozygous TT genotype of XRCC5 (1401 G>T, rs828907) $\left(p=0.038^{*}\right.$, OR $3.064,95 \%$ CI 1.063-8.835) and Heterozygous TC genotype of TGF $\beta 1$ (869 T>C, rs1982073) $\left(p=0.020^{*}\right.$, OR 4.606, $95 \%$ CI 1.272-16.674) along with history of alcohol intake $\left(p=0.023^{\star}\right.$, OR $3.584,95 \%$ CI 1.189-10.803).

On multivariate logistic regression analysis, only the first three polymorphisms remained statistically significant and were independent predictors of the risk of severe subcutaneous fibrosis; Homozygous CC genotype of XRCC3 (722 C>T, rs861539) $\left(p=0.013^{*}\right.$, OR $2.350,95 \%$ CI 1.089-5.382), Homozygous AA genotype of ERCC4Ex8 (1244 G>A, rs1800067) $\left(p=0.001^{\star *}\right.$, OR 
TABLE 2 | SNP distribution and toxicity status.

\begin{tabular}{|c|c|c|}
\hline \multirow{2}{*}{$\begin{array}{l}\text { SNP distribution } \\
\text { XRCC1 (rs25487) }\end{array}$} & \multicolumn{2}{|c|}{ Fibrosis N (\%) } \\
\hline & $\leq$ Grade 2 & Grade 3 \\
\hline Homozygous GG & $53(73.6 \%)$ & 19 (26.4\%) \\
\hline Heterozygous GA & $65(83.3 \%)$ & $13(16.7 \%)$ \\
\hline Homozygous AA & $25(86.2 \%)$ & $4(13.8 \%)$ \\
\hline$p$ value (Chi-square) & \multicolumn{2}{|c|}{0.112} \\
\hline XRCC3 (rs861539) & $\leq$ Grade 2 & Grade 3 \\
\hline Homozygous CC & 75 (76.5\%) & 23 (23.5\%) \\
\hline Heterozygous CT & $51(82.3 \%)$ & $11(17.7 \%)$ \\
\hline Homozygous Tा & $2(10.5 \%)$ & 17 (89.5\%) \\
\hline$p$ value & \multicolumn{2}{|c|}{$0.012^{*}$} \\
\hline XRCC4 (rs6869366) & $\leq$ Grade 2 & Grade 3 \\
\hline Homozygous $\pi$ & 105 (82.7\%) & 22 (17.3\%) \\
\hline Heterozygous TG & $32(71.1 \%)$ & 13 (28.9\%) \\
\hline Homozygous GG & $3(85.7 \%)$ & $1(14.3 \%)$ \\
\hline$p$ value & \multicolumn{2}{|c|}{0.570} \\
\hline XRCC5 (rs828907) & $\leq$ Grade 2 & Grade 3 \\
\hline Homozygous GG & $42(79.2 \%)$ & 11 (20.8\%) \\
\hline Heterozygous GT & $71(82.6 \%)$ & 15 (17.4\%) \\
\hline Homozygous TT & $10(25 \%)$ & 30 (75\%) \\
\hline$p$ value & \multicolumn{2}{|c|}{$0.046^{*}$} \\
\hline XRCC6 (rs2267437) & $\leq$ Grade 2 & Grade 3 \\
\hline Homozygous CC & 69 (79.3\%) & $18(20.7 \%)$ \\
\hline Heterozygous CG & $57(81.4 \%)$ & $13(18.6 \%)$ \\
\hline Homozygous GG & $17(77.3 \%)$ & $5(22.7 \%)$ \\
\hline$p$ value & \multicolumn{2}{|c|}{0.885} \\
\hline ERCC4 (rs1799801) & $\leq$ Grade 2 & Grade 3 \\
\hline Homozygous $\pi$ & $34(75.6 \%)$ & 11 (24.4\%) \\
\hline Heterozygous TC & $59(79.7 \%)$ & 15 (20.3\%) \\
\hline Homozygous CC & $50(83.3 \%)$ & $10(16.7)$ \\
\hline$p$ value & \multicolumn{2}{|c|}{0.133} \\
\hline ERCC4 (rs1800067) & $\leq$ Grade 2 & Grade 3 \\
\hline Homozygous GG & 127 (81.4\%) & $29(18.6 \%)$ \\
\hline Heterozygous GA & $16(80 \%)$ & 4 (20\%) \\
\hline Homozygous AA & $0(0 \%)$ & $3(100 \%)$ \\
\hline$p$ value & \multicolumn{2}{|c|}{$0.003^{* *}$} \\
\hline RAD51 (rs1801321) & $\leq$ Grade 2 & Grade 3 \\
\hline Homozygous GG & 55 (83.3\%) & $11(16.7 \%)$ \\
\hline Heterozygous GT & $43(78.2 \%)$ & 12 (21.8\%) \\
\hline Homozygous Tा & $45(77.6 \%)$ & 13 (22.4\%) \\
\hline$p$ value & \multicolumn{2}{|c|}{0.747} \\
\hline TgF $\beta 1$ (rs1982073) & $\leq$ Grade 2 & Grade 3 \\
\hline Homozygous $T ा$ & $134(81.7 \%)$ & 30 (18.3\%) \\
\hline Heterozygous TC & $6(40 \%)$ & $9(60 \%)$ \\
\hline Homozygous CC & $0(0 \%)$ & $0(0 \%)$ \\
\hline$p$ value & \multicolumn{2}{|c|}{$0.045^{*}$} \\
\hline TgFß1 (rs1800469) & $\leq$ Grade 2 & Grade 3 \\
\hline Homozygous GG & $128(80 \%)$ & $32(20 \%)$ \\
\hline Heterozygous GT & $11(73.3 \%)$ & $4(26.7 \%)$ \\
\hline Homozygous $\pi$ & $4(100 \%)$ & $0(0 \%)$ \\
\hline$p$ value & \multicolumn{2}{|c|}{0.246} \\
\hline
\end{tabular}

Bold values indicate the statistically significant $p$ values on Chi-square/Fischer exact test; ${ }^{*} p$ values $\leq 0.05,{ }^{* *} p$ values $<0.01$.

11.626, 95\% CI 2.490-275.901) and Homozygous TT genotype of XRCC5 (1401 G>T, rs828907) $\left(p=0.020^{*}\right.$, OR 2.188, 95\% CI 1.6527.334) (Table 3).

\section{Haplotype Analysis}

Presence of either of the haplotypes ie. variant Homozygous AA genotype of ERCC4Ex8 (1244 G>A, rs1800067) or Homozygous TT genotype of XRCC5 (1401 G>T, rs828907) was associated with a significantly increased risk of severe fibrosis $(\boldsymbol{p}=\mathbf{0 . 0 5 0}$, OR $2.837,95 \%$ CI 1.317-5.212) when compared to carriers of the
TABLE 3 | Multivariate analysis of genotypic variables and clinical characteristics with risk of subcutaneous fibrosis.

\begin{tabular}{|c|c|c|}
\hline Genotypic/Clinical Variable & $\begin{array}{l}\text { Multivariate Odds Ratio } \\
\qquad(95 \% \mathrm{Cl})\end{array}$ & $\begin{array}{c}p \\
\text { Value }\end{array}$ \\
\hline $\begin{array}{l}\text { Homozygous AA genotype of ERCC4 Ex8 } \\
1244 G>A\end{array}$ & $2.350(1.089-5.382)$ & $0.001^{* *}$ \\
\hline $\begin{array}{l}\text { Homozygous CC genotype of XRCC3 } \\
722 \mathrm{C}>\mathrm{T}\end{array}$ & $11.626(2.490-275.901)$ & $0.013^{*}$ \\
\hline $\begin{array}{l}\text { Homozygous TT genotype of XRCC5 } \\
1401 G>T\end{array}$ & $2.188(1.652-7.334)$ & $0.020^{*}$ \\
\hline $\begin{array}{l}\text { Heterozygous CC genotype of TGF } \beta 1 \text { (869 } \\
T>C, \text { rs1982073) }\end{array}$ & $4.368(0.976-19.540)$ & 0.054 \\
\hline Alcohol intake & $3.209(0.966-10.668)$ & 0.057 \\
\hline
\end{tabular}

wild type or heterozygous variants. A cumulative increased risk of developing severe fibrosis was observed in the presence of both haplotypes ( $p=0.010^{*}$, OR 26.340, 95\% CI 4.014-76.568).

\section{DISCUSSION}

This study was conducted to investigate the impact of genetic variants of DNA repair and pro-fibrotic pathway genes on the development of severe radiation-induced subcutaneous fibrosis in patients of OPC.

SNPs of three DNA repair genes; XRCC3 (722 C>T, rs861539), ERCC4Ex8 (1244 G>A, rs1800067) and XRCC5 (1401 G>T, rs828907) were shown to significantly increase the risk of developing severe radiation-induced subcutaneous fibrosis. In addition, haplotypes of ERCC4Ex8 (1244 G>A, rs1800067) and XRCC5 (1401 G>T, rs828907) polymorphisms had a highly significant combined predictive effect on the risk of severe fibrosis.

XRCC3 polymorphisms have been extensively tested for their association with radiotoxicities in a variety of cancers. De Ruyck et al. analyzed XRCC3 polymorphisms in cervical cancer samples and concluded that SNPs of XRCC3 are associated with an increased risk of late toxic effects after radiation (34). Andreassen et al. reported that Thr/Thr genotype in XRCC3 codon 241 correlated with an increased risk of subcutaneous fibrosis as well as telangiectasia in breast cancer (33). Another study by Damaraju et al. found significant univariate associations between late rectal or bladder toxicity and XRCC3 SNPs (38). XRCC3 $722 \mathrm{C}>\mathrm{T}$ allele has also been associated with an increased risk of radiation-induced late xerostomia in nasopharyngeal cancer patients (25). The association of this polymorphism with radiation-induced subcutaneous fibrosis in HNCs has not been demonstrated till date.

XRCC5 rs1051677 (T>C) C allele has been shown to be associated with severe subcutaneous fibrosis in patients of nasopharyngeal carcinoma in a study by Alsbeih et al, though the authors could not replicate these findings in their multivariate analysis (29). In a study by Yin et al, women with AG/AA genotypes of XRCC5 rs3835 (G>A) were at increased risk of severe radiation pneumonitis (40). 
The association of SNPs of ERCC4 with the risk of radiationinduced fibrosis has not been reported previously. Although, studies have investigated its correlation with other radiotoxicity end-points such as dysphagia and feeding tube dependence in patients of HNC. In a study of 130 patients of OPC treated with radiotherapy, Kornguth et al. studied the association of two SNPs in XPF/ERCC4 and long-term use of percutaneous feeding tube. The Homozygous AA genotype of ERCC4 Ex8 1244G>A was associated with a reduced need for feeding tube, but this association was not statistically significant. Although the wild Homozygous TT genotype of the second SNP ERCC4 Ex11 2505T $>$ C showed a protective effect and was significantly associated with decreased long-term gastrostomy tube dependence (27). In our study the variant allele of ERCC4 Exon 8 was associated with an increased risk of severe subcutaneous fibrosis.

The heterozygous TC genotype of TGF $\beta 1$ (869 T>C, rs1982073) correlated with severe subcutaneous fibrosis on univariate analysis. However, no significant association could be seen on multivariate analysis. In a study by Alsbeih et al. on patients of nasopharyngeal carcinoma, it was seen that the wildtype allele of TGF $\beta 869$ T>C contributed to the severity of radiation-induced subcutaneous fibrosis (29). In sites other than head and neck, TGF $\beta 869$ T>C polymorphism has shown significant associations with the risk of radiation-induced fibrosis in patients of breast cancer after breast conserving surgery (41). Other published studies suggested that the variant C allele was the risk factor $(33,42)$.

Of all patient specific clinical factors that were analyzed, history of alcohol intake was significantly associated with the risk of developing severe subcutaneous fibrosis. However, it failed to show significance in multivariate analysis. Although there is convincing evidence that acetaldehyde, the first metabolite produced during alcohol degradation, is responsible for the carcinogenic effect of ethanol owing to its multiple mutagenic effects on DNA (43), no association with risk of radiation related toxicities has been demonstrated till date.

Our findings showed significant association between SNPs of DNA repair genes and risk of severe subcutaneous fibrosis in patients of OPC treated with radiotherapy. These are encouraging results and suggest that genetic variations contribute to the severity of normal tissue toxicities after radiotherapy. More importantly, we also performed a haplotype association analysis of two polymorphisms for predicting the combined risk of severe subcutaneous fibrosis. Haplotype-based analysis may offer better genetic information and help improve the detection of causal genetic variants when compared with single SNP-based analysis (44).

$\mathrm{OPC}$ is a heterogeneous population with varying natural history and disease course. To ensure homogeneity in radiation portals and eliminate any confounding related to previous surgical resection or administration of concurrent chemotherapy, we included only those patients of OPC who were being treated with definitive radiotherapy. Most patients had advanced disease at presentation or bulky midline tumours involving the base of tongue and soft palate. Hence, bilateral neck irradiation was given in all cases, removing any confounding due to differences in field size $(45,46)$.
Though tumor HPV status is a strong and independent prognostic factor for survival among patients with OPC (47), it was not analyzed in our study. This was due to lack of adequate infrastructure along with lower HPV prevalence amidst the high tobacco burden in the country $(48,49)$. Likewise, approximately $90 \%$ of patients in our study population were smokers.

It is acknowledged that conformal techniques were not used in this study owing to the enormous patient load in a limited resource setting $(3,4)$. The number of patients with OPC treated at our center in the previous five years (2015-2019) ranged from 300-350 per year, as per the Hospital Based Cancer Registry data.

A candidate gene approach was used and only a limited number of SNPs were selected for study. SNPs represent a majority of heritable genetic variations, are often inherited together and multiple such variations may affect radiation response. Thus, the selection of candidate genes is a critical step in determining the genetic basis of normal tissue radiosensitivity. In this study, we have opted to give a high priority to SNPs that have been demonstrated to significantly influence biological processes such as DNA repair, which continue to be the most studied pathways for HNC outcomes (50).

The major limitation of a candidate gene approach is that it requires a prior knowledge of the gene function and previously unknown genetic variants involved in the phenotype are missed. Moreover, candidate gene studies are usually underpowered to detect the small effect sizes that are attributed to SNPs. It is critical to employ a genome-wide approach to overcome this limitation. Genome Wide Association Studies (GWAS) allow us to map the entire genome for the presence of genetic variants that could possibly have a significant impact on normal tissue radiosensivity. GWAS offer the advantage of studying all SNPs, including those in regulatory regions whose function is not fully understood. However, these studies require large sample sizes to be considered reliable and may detect many false positive SNPs that are unimportant in relevant biological processes. Replication studies should be carried out to distinguish the true positive SNPs that may have a role in influencing radiosensitivity (17). Also, other pathways that could be hypothetically involved in normal tissue radiosensitivity, such as oxidative stress response, activation of cell cycle checkpoints, inflammation and apoptosis are yet to be thoroughly investigated (29).

The results from this study, upon further validation would enable us to identify patients who are genetically predisposed to the development of severe radiation-induced subcutaneous fibrosis. We propose to incorporate these genetic markers into predictive models of normal tissue toxicity in combination with patient and clinical factors. Such a profile could divide patients into subgroups with different probabilities of developing toxicity, to permit irradiation up to the normal tissue tolerance for each subgroup. These 'at risk' patient groups could then be offered treatment with individualized protocols and with more conformal radiotherapy techniques like Intensity Modulated Radiation Therapy (IMRT). This is expected to aid in judicious allocation of the limited available resources in developing countries and also allow improved compliance with standard treatment schedules leading to better outcome with least morbidity. 


\section{CONCLUSION}

The ultimate goal of radiogenomics research is to tailor radiation therapy protocols based on a combination of genetic, clinical and treatment related factors, in order to optimize tumour control while causing minimal normal tissue damage. In the present study, we demonstrated significant associations between SNPs of DNA repair genes and severe radiation-induced subcutaneous fibrosis in oropharyngeal carcinoma. A multivariate predictive model was developed and combination of haplotypes were identified to characterize patients at high risk of severe subcutaneous fibrosis. The identified predictors of radiosensitivity are aimed to ultimately contribute to an algorithm for guiding therapy tailored to the patient's risk and benefit profile.

\section{DATA AVAILABILITY STATEMENT}

The original contributions presented in the study are included in the article/Supplementary Material. Further inquiries can be directed to the corresponding author.

\section{ETHICS STATEMENT}

The studies involving human participants were reviewed and approved by Institute Ethics Committee, Post Graduate Institute of Medical Education and Research, Chandigarh, India. The patients/participants provided their written informed consent to participate in this study.

\section{REFERENCES}

1. Mathur P, Sathishkumar K, Chaturvedi M, Das P, Sudarshan KL, Santhappan S, et al. Cancer Statistics, 2020: Report From National Cancer Registry Programme, India. JCO Global Oncol (2020) 6:1063-75. doi: 10.1200/GO.20.00122

2. Pignon JP, le Maitre A, Maillard E, Bourhis J, Group M-NC. Meta-Analysis of Chemotherapy in Head and Neck Cancer (MACH-NC): An Update on 93 Randomised Trials and 17,346 Patients. Radiother Oncol (2009) 92(1):4-14. doi: 10.1016/j.radonc.2009.04.014

3. Ghoshal S, Goda JS, Mallick I, Kehwar TS, Sharma SC. Concomitant Boost Radiotherapy Compared With Conventional Radiotherapy in Squamous Cell Carcinoma of the Head and Neck-a Phase III Trial From a Single Institution in India. Clin Oncol (R Coll Radiol). (2008) 20(3):212-20. doi: 10.1016/j.clon. 2008.01.011

4. Rishi A, Ghoshal S, Verma R, Oinam AS, Patil VM, Mohinder R, et al. Comparison of Concomitant Boost Radiotherapy Against Concurrent Chemoradiation in Locally Advanced Oropharyngeal Cancers: A Phase III Randomised Trial. Radiother Oncol (2013) 107(3):317-24. doi: 10.1016/j.radonc.2013.05.016

5. Corry J, Peters LJ, Rischin D. Optimising the Therapeutic Ratio in Head and Neck Cancer. Lancet Oncol (2010) 11(3):287-91. doi: 10.1016/S1470-2045(09)70384-5

6. Prabhash K, Babu G, Chaturvedi P, Kuriakose M, Birur P, Anand AK, et al. Indian Clinical Practice Consensus Guidelines for the Management of Squamous Cell Carcinoma of Head and Neck. Indian J Cancer (2020) 57 (Supplement):S1-s5. doi: 10.4103/0019-509X.278971

7. Gupta A, Mathew D, Ghoshal S, Pal A. XRCC1 (Rs25487) Polymorphism is Associated With Severe Oral Mucositis and Poor Treatment Response After Radiotherapy for Oropharyngeal Carcinoma. Oral Cancer (2019) 3(3):37-47. doi: 10.1007/s41548-019-00021-x

8. Gupta T, Kannan S, Ghosh-Laskar S, Agarwal JP. Systematic Review and MetaAnalyses of Intensity-Modulated Radiation Therapy Versus Conventional TwoDimensional and/or or Three-Dimensional Radiotherapy in Curative-Intent

\section{AUTHORS CONTRIBUTIONS}

Study concept: SG and AP. Study design: AP. Data acquisition: AG and DM. Quality control of data and algorithms: AG, DM, and AP. Data analysis and interpretation: DM and AP. Statistical analysis: DM and AP. Manuscript preparation: AG and SB Manuscript editing: SB, SG, and AP. All authors contributed to the article and approved the submitted version.

\section{FUNDING}

The study was partially funded by Special Research Grant by PGIMER, Chandigarh.

\section{ACKNOWLEDGMENTS}

We acknowledge Indian Council of Medical Research (ICMR), New Delhi for Fellowship to DM (Grant No: 3/1/1/JRF-2014/ HRD-09 (32539)) and partial support for the research work and fellowship to SB (Grant No 52/04/2019-BIO/BMS). We also thank Dr Nishant Jindal for his contribution to statistical analysis.

\section{SUPPLEMENTARY MATERIAL}

The Supplementary Material for this article can be found online at: https://www.frontiersin.org/articles/10.3389/fonc.2021.652049/ full\#supplementary-material

Management of Head and Neck Squamous Cell Carcinoma. PloS One (2018) 13(7):e0200137. doi: 10.1371/journal.pone.0200137

9. Trotti A, Bellm LA, Epstein JB, Frame D, Fuchs HJ, Gwede CK, et al. Mucositis Incidence, Severity and Associated Outcomes in Patients With Head and Neck Cancer Receiving Radiotherapy With or Without Chemotherapy: A Systematic Literature Review. Radiother Oncol (2003) 66(3):253-62. doi: 10.1016/s01678140(02)00404-8

10. Machtay M, Moughan J, Trotti A, Garden AS, Weber RS, Cooper JS, et al. Factors Associated With Severe Late Toxicity After Concurrent Chemoradiation for Locally Advanced Head and Neck Cancer: An RTOG Analysis. JClin Oncol(2008) 26(21):3582-9. doi: 10.1200/JCO.2007.14.8841

11. Deng J, Wulff-Burchfield EM, Murphy BA. Late Soft Tissue Complications of Head and Neck Cancer Therapy: Lymphedema and Fibrosis. J Natl Cancer Inst Monogr (2019) 2019(53):63-71. doi: 10.1093/jncimonographs/lgz005

12. Straub JM, New J, Hamilton CD, Lominska C, Shnayder Y, Thomas SM. Radiation-Induced Fibrosis: Mechanisms and Implications for Therapy. J Cancer Res Clin Oncol (2015) 141(11):1985-94. doi: 10.1007/s00432-015-1974-6

13. Ridner SH, Dietrich MS, Niermann K, Cmelak A, Mannion K, Murphy B. A Prospective Study of the Lymphedema and Fibrosis Continuum in Patients With Head and Neck Cancer. Lymphatic Res Biol (2016) 14(4):198-205. doi: 10.1089/lrb.2016.0001

14. Parliament MB, Murray D. Single Nucleotide Polymorphisms of DNA Repair Genes as Predictors of Radioresponse. Semin Radiat Oncol (2010) 20(4):23240. doi: 10.1016/j.semradonc.2010.05.003

15. Madhusudan S, Middleton MR. The Emerging Role of DNA Repair Proteins as Predictive, Prognostic and Therapeutic Targets in Cancer. Cancer Treat Rev (2005) 31(8):603-17. doi: 10.1016/j.ctrv.2005.09.006

16. Barnett GC, Kerns SL, Noble DJ, Dunning AM, West CM, Burnet NG. Incorporating Genetic Biomarkers Into Predictive Models of Normal Tissue Toxicity. Clin Oncol (R Coll Radiol) (2015) 27(10):579-87. doi: 10.1016/j.clon. 2015.06 .013 
17. Brothwell MRS, West CM, Dunning AM, Burnet NG, Barnett GC. Radiogenomics in the Era of Advanced Radiotherapy. Clin Oncol ( $R$ Coll Radiol). (2019) 31(5):319-25. doi: 10.1016/j.clon.2019.02.006

18. Bourguignon MH, Gisone PA, Perez MR, Michelin S, Dubner D, Giorgio MD, et al. Genetic and Epigenetic Features in Radiation Sensitivity. Part II: Implications for Clinical Practice and Radiation Protection. Eur J Nucl Med Mol Imaging (2005) 32(3):351-68. doi: 10.1007/s00259-004-1731-6

19. Chistiakov DA, Voronova NV, Chistiakov PA. Genetic Variations in DNA Repair Genes, Radiosensitivity to Cancer and Susceptibility to Acute Tissue Reactions in Radiotherapy-Treated Cancer Patients. Acta Oncol (2008) 47 (5):809-24. doi: 10.1080/02841860801885969

20. Jackson SP. Sensing and Repairing DNA Double-Strand Breaks. Carcinogenesis (2002) 23(5):687-96. doi: 10.1093/carcin/23.5.687

21. Alsbeih G, Al-Harbi N, Al-Hadyan K, El-Sebaie M, Al-Rajhi N. Association Between Normal Tissue Complications After Radiotherapy and Polymorphic Variations in TGFB1 and XRCC1 Genes. Radiat Res (2010) 173(4):505-11. doi: $10.1667 / R R 1769.1$

22. Yang Z, Liu Z. Potential Functional Variants in DNA Repair Genes are Associated With Efficacy and Toxicity of Radiotherapy in Patients With Non-Small-Cell Lung Cancer. J Oncol (2020) 2020:3132786. doi: 10.1155/2020/3132786

23. Andreassen CN, Alsner J, Overgaard M, Sorensen FB, Overgaard J. Risk of Radiation-Induced Subcutaneous Fibrosis in Relation to Single Nucleotide Polymorphisms in TGFB1, SOD2, XRCC1, XRCC3, APEX and ATM-a Study Based on DNA From Formalin Fixed Paraffin Embedded Tissue Samples. Int J Radiat Biol (2006) 82(8):577-86. doi: 10.1080/09553000600876637

24. Araujo FD, Pierce AJ, Stark JM, Jasin M. Variant XRCC3 Implicated in Cancer is Functional in Homology-Directed Repair of Double-Strand Breaks. Oncogene. (2002) 21(26):4176-80. doi: 10.1038/sj.onc.1205539

25. Zou Y, Song T, Yu W, Zhao R, Wang Y, Xie R, et al. XRCC3 Polymorphisms are Associated With the Risk of Developing Radiation-Induced Late Xerostomia in Nasopharyngeal Carcinoma Patients Treated With Intensity Modulation Radiated Therapy. Jpn J Clin Oncol (2014) 44(3):241-8. doi: 10.1093/jjco/hyt202

26. Manandhar M, Boulware KS, Wood RD. The ERCC1 and ERCC4 (XPF) Genes and Gene Products. Gene. (2015) 569(2):153-61. doi: 10.1016/j.gene.2015.06.026

27. Kornguth DG, Garden AS, Zheng Y, Dahlstrom KR, Wei Q, Sturgis EM. Gastrostomy in Oropharyngeal Cancer Patients With ERCC4 (XPF) Germline Variants. Int J Radiat Oncol Biol Phys (2005) 62(3):665-71. doi: 10.1016/j.ijrobp.2004.11.026

28. Al-Hadyan KS, Al-Harbi NM, Al-Qahtani SS, Alsbeih GA. Involvement of Single-Nucleotide Polymorphisms in Predisposition to Head and Neck Cancer in Saudi Arabia. Genet Test Mol Biomarkers (2012) 16(2):95-101. doi: 10.1089/gtmb.2011.0126

29. Alsbeih G, El-Sebaie M, Al-Harbi N, Al-Hadyan K, Shoukri M, Al-Rajhi N. Snps in Genes Implicated in Radiation Response are Associated With Radiotoxicity and Evoke Roles as Predictive and Prognostic Biomarkers. Radiat Oncol (2013) 8:125. doi: 10.1186/1748-717X-8-125

30. Wiese C, Collins DW, Albala JS, Thompson LH, Kronenberg A, Schild D. Interactions Involving the Rad51 Paralogs Rad51C and XRCC3 in Human Cells. Nucleic Acids Res (2002) 30(4):1001-8. doi: 10.1093/nar/30.4.1001

31. Li L, Zhang X, Zhang ZT. Genetic Polymorphisms in the RAD51 Gene With a Risk of Head and Neck Cancer and Esophageal Cancer: A Meta-Analysis. Int J Genomics (2019) 2019:2789035. doi: 10.1155/2019/2789035

32. Pratesi N, Mangoni M, Mancini I, Paiar F, Simi L, Livi L, et al. Association Between Single Nucleotide Polymorphisms in the XRCC1 and RAD51 Genes and Clinical Radiosensitivity in Head and Neck Cancer. Radiother Oncol (2011) 99(3):356-61. doi: 10.1016/j.radonc.2011.05.062

33. Andreassen CN, Alsner J, Overgaard M, Overgaard J. Prediction of Normal Tissue Radiosensitivity From Polymorphisms in Candidate Genes. Radiother Oncol (2003) 69(2):127-35. doi: 10.1016/j.radonc.2003.09.010

34. De Ruyck K, Van Eijkeren M, Claes K, Bacher K, Vral A, De Neve W, et al. Tgfbeta1 Polymorphisms and Late Clinical Radiosensitivity in Patients Treated for Gynecologic Tumors. Int J Radiat Oncol Biol Phys (2006) 65(4):1240-8. doi: 10.1016/j.ijrobp.2006.03.047

35. Andreassen CN, Overgaard J, Alsner J, Overgaard M, Herskind C, Cesaretti JA, et al. ATM Sequence Variants and Risk of Radiation-Induced Subcutaneous Fibrosis After Postmastectomy Radiotherapy. Int J Radiat Oncol Biol Phys (2006) 64(3):776-83. doi: 10.1016/j.ijrobp.2005.09.014
36. Burri RJ, Stock RG, Cesaretti JA, Atencio DP, Peters S, Peters CA, et al. Association of Single Nucleotide Polymorphisms in SOD2, XRCC1 and XRCC3 With Susceptibility for the Development of Adverse Effects Resulting From Radiotherapy for Prostate Cancer. Radiat Res (2008) 170(1):49-59. doi: 10.1667/RR1219.1

37. Cesaretti JA, Stock RG, Lehrer S, Atencio DA, Bernstein JL, Stone NN, et al. ATM Sequence Variants are Predictive of Adverse Radiotherapy Response Among Patients Treated for Prostate Cancer. Int J Radiat Oncol Biol Phys (2005) 61(1):196-202. doi: 10.1016/j.ijrobp.2004.09.031

38. Damaraju S, Murray D, Dufour J, Carandang D, Myrehaug S, Fallone G, et al. Association of DNA Repair and Steroid Metabolism Gene Polymorphisms With Clinical Late Toxicity in Patients Treated With Conformal Radiotherapy for Prostate Cancer. Clin Cancer Res (2006) 12(8):2545-54. doi: 10.1158/10780432.CCR-05-2703

39. Cox JD, Stetz J, Pajak TF. Toxicity Criteria of the Radiation Therapy Oncology Group (RTOG) and the European Organization for Research and Treatment of Cancer (EORTC). Int J Radiat Oncol Biol Phys (1995) 31(5):1341-6. doi: 10.1016/0360-3016(95)00060-c

40. Yin M, Liao Z, Liu Z, Wang LE, O’Reilly M, Gomez D, et al. Genetic Variants of the Nonhomologous End Joining Gene LIG4 and Severe Radiation Pneumonitis in Nonsmall Cell Lung Cancer Patients Treated With Definitive Radiotherapy. Cancer (2012) 118(2):528-35. doi: 10.1002/cncr.26214

41. Terrazzino S, La Mattina P, Gambaro G, Masini L, Franco P, Canonico PL, et al. Common Variants of GSTP1, GSTA1, and Tgf $\beta 1$ are Associated With the Risk of Radiation-Induced Fibrosis in Breast Cancer Patients. Int J Radiat Oncol Biol Phys (2012) 83(2):504-11. doi: 10.1016/j.ijrobp.2011.06.2012

42. Andreassen CN, Alsner J, Overgaard J, Herskind C, Haviland J, Owen R, et al. TGFB1 Polymorphisms are Associated With Risk of Late Normal Tissue Complications in the Breast After Radiotherapy for Early Breast Cancer. Radiother Oncol (2005) 75(1):18-21. doi: 10.1016/j.radonc.2004.12.012

43. Seitz HK, Stickel F. Molecular Mechanisms of Alcohol-Mediated Carcinogenesis. Nat Rev Cancer (2007) 7(8):599-612. doi: 10.1038/nrc2191

44. Crawford DC, Nickerson DA. Definition and Clinical Importance of Haplotypes. Annu Rev Med (2005) 56(1):303-20. doi: 10.1146/annurev.med.56.082103.104540

45. Paleri V, Urbano TG, Mehanna H, Repanos C, Lancaster J, Roques T, et al. Management of Neck Metastases in Head and Neck Cancer: United Kingdom National Multidisciplinary Guidelines. J Laryngol Otol (2016) 130(S2):S161S9. doi: 10.1017/S002221511600058X

46. Mendenhall WM, Million RR. Elective Neck Irradiation for Squamous Cell Carcinoma of the Head and Neck: Analysis of Time-Dose Factors and Causes of Failure. Int J Radiat Oncol Biol Phys (1986) 12(5):741-6. doi: 10.1016/03603016(86)90031-3

47. Ang KK, Harris J, Wheeler R, Weber R, Rosenthal DI, Nguyen-Tan PF, et al. Human Papillomavirus and Survival of Patients With Oropharyngeal Cancer. N Engl J Med (2010) 363(1):24-35. doi: 10.1056/NEJMoa0912217

48. Murthy V, Calcuttawala A, Chadha K, d'Cruz A, Krishnamurthy A, Mallick I, et al. Human Papillomavirus in Head and Neck Cancer in India: Current Status and Consensus Recommendations. South Asian J Cancer (2017) 6(3):93-8. doi: 10.4103/sajc.sajc_96_17

49. Murthy V, Swain M, Teni T, Pawar S, Kalkar P, Patil A, et al. Human Papillomavirus/ P16 Positive Head and Neck Cancer in India: Prevalence, Clinical Impact, and Influence of Tobacco Use. Indian J Cancer (2016) 53(3):387-93. doi: 10.4103/0019509X.200668

50. Hopkins J, Cescon DW, Tse D, Bradbury P, Xu W, Ma C, et al. Genetic Polymorphisms and Head and Neck Cancer Outcomes: A Review. Cancer Epidemiol Biomarkers Prev (2008) 17(3):490-9. doi: 10.1158/1055-9965.EPI-07-2714

Conflict of Interest: The authors declare that the research was conducted in the absence of any commercial or financial relationships that could be construed as a potential conflict of interest.

Copyright (C) 2021 Gupta, Mathew, Bhat, Ghoshal and Pal. This is an open-access article distributed under the terms of the Creative Commons Attribution License (CC BY). The use, distribution or reproduction in other forums is permitted, provided the original author(s) and the copyright owner(s) are credited and that the original publication in this journal is cited, in accordance with accepted academic practice. No use, distribution or reproduction is permitted which does not comply with these terms. 